\title{
Über einige Eigenschaften gelöster Fadenmoleküle, I. Mitteilung.
}

\section{(Zur Theorie der Lösungen hochpolymerer Substanzen II)}

\author{
Von Arnold Münster
}

\author{
Aus dem Chemischen Institut der Universität Heidelberg \\ (7.. Naturforschg. 1, 311-320 [1946]; eingegangen am 23. März 1946)
}

1. Die empirischen thermodynamischen Eigenschaften gelöster Fadenmoleküle und die bisherigen Versuche zu ihrer Deutung werden besprochen.

2. Es wird eine statistische Theorie der polydispersen athermischen Lösung entwickelt. Die Berechnung des Kombinationsfaktors beruht auf dem Ansatz, daß die Zahl der Komplexionen durch die Zahl $\Lambda$ der möglichen Anordnungen für die Fadenmoleküle, die als virtuelle Fadenmoleküle bezeichnet werden, bestimmt ist.

3. Die Abhängigkeit der Größe $\Lambda$ von der Gestalt, Größe und Beweglichkeit der Fadenmoleküle wird diskutiert. Der absolute Betrag der Abweichung von der idealen Lösung wird durch den Störungsparameter $\alpha$ bestimmt. Seine Beziehung zur Größe und Beweglichkeit der Fadenmoleküle und zur Struktur der Flüssigkeit wird untersucht.

4. Es werden Formeln für die chemischen Potentiale, die Verdünnungsentropie und die Aktivität des Lösungsmittels abgeleitet.

5. Die Formeln für die Verdünnungsentropie und die Aktivität des Lösungsmittels werden mit verschiedenen Messungsergebnissen verglichen. Das Verhalten näherungsweise athermischer Lösungen wird durch die Theorie befriedigend wiedergegeben. Daraus kann geschlossen werden, daß die charakteristischen thermodynamischen Eigenschaften gelöster Fadenmoleküle in erster Linie durch ihre Größe, Gestalt und Beweglichkeit bestimmt werden und sich nicht auf die ideale Lösung zurückführen lassen. Im besonderen ergibt sich, daß die Moleküle der Polyprene nur eine verhältnismäßig geringe Beweglichkeit in der Lösung besitzen.

$I^{n}$ $\mathrm{n}$ einer früheren Arbeit ${ }^{1}$ sind die Grundlagen einer statistischen Theorie der Lösungen hochpolymerer Substanzen entwickelt worden. Es konnte gezeigt werden, daß die charakteristischen osmotischen Eigenschaften dieser Lösungen sich aus dem Größenunterschied der Moleküle des Lösungsmittels (LM) und der gelösten Substanzen ableiten lassen, ohne daß es im Prinzip weiterer Annahmen über energetische Wechselwirkung (Solvatation) bedarf. In den folgenden Untersuchungen wird die Theorie auf einige Probleme angewandt, welche bei den Lösungen der Fadenmoleküle (FM) auftreten. Dazu ist es notwendig, auch die Grundgleichungen noch einmal in verallgemeinerter Form zu entwickeln.

I. Empirische Eigenschaften gelöster FM; allgemeine Gesichtspunkte zu ihrer

\section{Deutung}

Bei der theoretischen Behandlung der Lösungen ist es zweckmäßig, von einem Modell auszugehen, welches man exakt durchrechnen kann. Durch den

$$
1 \text { A. Münster, Kolloid-Z. 105, } 1 \text { [1943]. }
$$

Vergleich seiner Eigenschaften mit denen der empirischen Lösungen wird es ermöglicht, auch die letzteren zu deuten und in günstigen Fällen quantitativ zu formulieren. Ein derartiges Modell ist die von $\mathrm{Lew}$ is ${ }^{2}$ in die Thermodynamik eingeführte ,ideale Lösung“. Sie ist thermodynamisch definiert durch die Beziehung

$$
d \mu_{2}=R T d \ln s_{2},
$$

wo $\mu_{2}$ das chemische Potential und $N_{2}$ der Molenbruch des LM ist. Da man nach Lewi ${ }^{2}$ allgemein für Lösungen setzen' kann

$$
d \mu_{2}=R T d \ln a_{2},
$$

wo $a_{2}$ die Aktivität des LM ist, kann die ideale Lösung bei geeigneter Wahl des Bezugszustandes für $a_{2}$ auch durch die Gleichung

definiert werden.

$$
a_{2} / N_{2}=1
$$

Auf Grund der thermodynamischen Beziehung $\mu_{2}-\mu_{02}=R T \ln a_{2}=H_{2}-H_{02}-T\left(s_{2}-s_{02}\right)$

${ }^{2}$ G. N. Lewis u. M. Randall, Thermodynamik, deutsch von O. Redlich, Wien 1927. 
$(\boldsymbol{H}=$ partialer Wärmeinhalt, $s=$ partiale Entropie; der Index ${ }_{0}$ bezieht sich auf das reine LM) läßt sich nach J. S. Hild ebrand ${ }^{3}$, Ch. G. B o is s o nna ${ }^{4}$, K. H. M eyer und A. J. A. van der $\mathrm{Wyk}^{5}$ eine weitergehende Systematik der Lösungen durchführen. Es werden folgende Typen unterschieden:

1. $H_{2}-H_{02}=0, s_{2}-s_{02}=-R \ln N_{2}$, (ideale Lösung)

2. $\mathrm{H}_{2}-\mathrm{H}_{02}==0, \mathrm{~s}_{2}-s_{02}=-R \ln \mathrm{N}_{2}$, (reguläre Lösung)

3. $\mathrm{H}_{2}-\mathrm{H}_{02}=0, s_{2}-s_{02}=-R \ln \mathrm{N}_{2}$, (athermische Lösung)

4. $H_{2}-H_{02}==0, s_{2}-s_{02}==-R \ln N_{2}$. (irreguläre Lösung)

Die Lösungen der FM zeigen Abweichungen vom Verhalten der idealen Lösung, welche durchweg der Beziehung

$$
a_{2} / N_{2}<1
$$

entsprechen $^{5}$. Sie lassen sich bereits bei niedrigmolekularen FM nachweisen ${ }^{4,6,7}$. Vor allem aber ist die Beziehung (5) charakteristisch für die Lösungen der hochpolymeren FM. Zwar zeigen auch sphärische hochpolymere Moleküle ein derartiges Verhalten (vergl. z. B. ${ }^{8}$ ), aber es ist hier viel weniger ausgeprägt als bei den FM.

Ein der Beziehung (5) entsprechendes Verhalten kann entweder durch die energetische Wechselwirkung der FM mit dem LM oder, unabhängig von dieser, durch ihre Größe, Gestalt und Beweglichkeit und schließlich durch beide Faktoren bedingt sein.

Für die Entscheidung der Frage, welche dieser Ursachen wirksam ist, kommen im wesentlichen folgende Möglichkeiten in Betracht:

1. Thermodynamische Untersuchungen auf Grund der obigen Systematik der Lösungen ${ }^{4,9-17}$.

"In zahlreichen Fällen ergab sich, unabhängig

3 J. Amer. chem. Soc. 51, 66 [1929].

4 Helv. chim. Acta 20, 768 [1937].

5 Helv. chim. Acta 23, 488 [1940].

6 J. N. Brönstedt u. P. Colmant, Z. physik. Chem. 168, 381 [1934].

7 K. H. Meyer u. R. Lühdemann, Helv. chim. Acta 18, 307 [1935].

8 G. S. Ad a ir, Proc. Roy. Soc. [London] (A) 120, 573 [1928].

9 G. V.Schulz, Z. physik. Chem. (A) 180, 1 [1937]. 10 G. V. Schulz, Z. physik. Chem. (B) 40, 319 [1938].

11 G. V.Schulz, Z. physik. Chem. (A) 184, 1 [1939]. von der positiven oder negativen Verdünnungswärme, eine gegenüber der idealen stark erhöhte Verdünnungsentropie. Zwar können Abweichungen der Entropie von dem für ideale Lösungen geltenden Wert auf Grund der thermodynamischen Beziehung

$$
\left[\frac{\partial\left(\mu_{2}-\mu_{02}\right)}{\partial T}\right]_{p, n_{1}, n_{2}}-\left(s_{2}-s_{02}\right)
$$

auch durch die Solvatation bedingt sein. K. H. Meyer, Wolff und Boissonnas ${ }^{15}$ sowie Wolff ${ }^{16}$ zeigten aber, daß sie auch bei den näherungsweise athermischen Lösungen von Kautschuk und Guttapercha in Toluol auftreten. Hier ist man daher gezwungen, von der Solvatation unabhängige Eigenschaften der FM zur Deutung der Beziehung (5) heranzuziehen. Daraus folgt, daß diese Effekte bei allen Lösungen der FM zu berücksichtigen sind. Unbeantwortet bleiben die Fragen nach ihrer molekularen Deutung und ihrer Abhängigkeit von den verschiedenen Variablen.

2. Beurteilung des Lösungszustandes auf Grund anderweitiger theoretischer oder experimenteller Ergebnisse.

R. Signer und H. Gro $ß^{18}$ zogen aus ihren Messungen an Polystyrolen mittels der Ultrazentrifuge den Schluß, daß starke konzentrationsabhängige Solvatation nicht vorliegen kann und der Zustand des Polystyrols in den meisten LM sehr ähnlich ist. $\mathrm{Zu}$ dem gleichen Ergebnis führt die Betrachtung der zwischenmolekularen Kräfte. Danach kann man das System Polystyrol - Toluol als näherungsweise athermisch betrachten.

\section{Ableitung spezieller, experimentell prüfbarer} Formeln.

G. V. Schulz ${ }^{19}$ hat eine Formel für den osmotischen Druck der Hochpolymeren abgeleitet auf Grund der Annahme, daß die Abweichungen von der idealen Lösung nur durch die Solvatation

12 G. V. Schulz, Z. Elektrochem. angew. physik. Chem. 45, 652 [1939].

${ }_{13}$ Ch. G. B o is s on nas u. K. H. Me yer, Helv. chim. Acta 20, 783 [1937].

14 O. Hagger u. A. J. A. van der Wyk, Helv. -chim. Acta 23, 484 [1940].

15 K. H. Me y e r, E. Wolf f u. Ch. G. B o is s onnas, Helv. chim. Acta 23, 430 [1940].

16 E. W olff, Helv. chim. Acta 23, 439 [1940].

17 G. Ge u. L. R. G. Tre lo ar, Trans. Faraday Soc. 38, 147 [1942].

18 Helv. chim. Acta 17, 59 [1934].

19 Z. physik. Chem. (A) 160, 409 [1932]. 
bestimmt werden. Diese Gleichung wird in dem experimentell prüfbaren Bereich gut bestätigt. Da ihre Ableitung jedoch gewissen Bedenken unterliegt, die an anderer Stelle erörtert werden sollen, kann sie nicht als Beweis für die Ausgangshypothese angesehen werden.

Die von Wo. Ostwald ${ }^{20}$ empirisch abgeleitete Formel für den osmotischen Druck der Hochpolymeren wurde von W. H a ll e r 21-23 sowohl aus der Beweglichkeit der FM wie aus der Solvatation begründet. Daraus ergibt sich bereits, daß auf diesem Wege keine Entscheidung der obigen Frage herbeizuführen ist. Die kinetische Theorie wurde überdies von $\mathrm{E} . \mathrm{H} \ddot{\mathrm{u}} \mathrm{ckel}^{24}$ eingehend kritisiert.

4. Allgemeine thermodynamische und statistische Überlegungen.

Von mehreren Autoren 25-31 wurde die Frage diskutiert, ob die Gesetze der idealen Lösung gelten können, wenn die Komponenten des Systems verschiedenes Molvolumen besitzen. Eine Rechnung von E.A.Guggenheim ${ }^{32}$ ergab, daß bei einer Verschiedenheit der Molvolumina von $25 \% \mathrm{Ab}$ weichungen vom $\mathrm{R}$ a oultschen Gesetz auftreten, diese aber meistens unter $1 \%$ liegen.

K. H. M e y e ${ }^{33}$ zeigte durch qualitative statistische Überlegungen, daß in einer Lösung von FM die Zahl der Komplexionen und damit die Entropie gegenüber der idealen Lösung modifiziert ist.

\section{Theorie der athermischen Lösung.}

Aus dem Vorstehenden ergeben sich zahlreiche Gründe für die Annahme, daß die thermodynamischen Eigenschaften gelöster FM jedenfalls nicht nur durch die Solvatation, sondern auch durch ihre Größe, Gestalt und vielleicht durch ihre Beweglichkeit bestimmt werden. Nicht beantwortet werden die Fragen, welche thermodynamischen Eigenschaften eine streng athermische Lösung be-

20 Kolloid-Z. 49, 60 [1929].

21 Kolloid-Z. 49, 74 [1929].

22 Kolloid-Z. 56, 257 [1931].

23 Kolloid-Z, 78, 341 [1937].

24 Z. Elektrochemie 42, 753 [1936].

25 R. P.Bell u. O.Gatty, Philos. Mag. J. Sci. (7) 19, 66 [1935].

26 R. P. B ell, Trans. Faraday Soc. 33, 496 [1937].

27 J. G. K i r k w o o d, Chem. Reviews 19, 274 [1936].

$28 \mathrm{~K}$. F r e d e $n \mathrm{~h}$ a g e $\mathrm{n}, \mathrm{Z}$. Elektrochem. angew. physik. Chem. 43, 28 [1937].

${ }_{29}$ K. Fredenhagen, Liebigs Ann. Chem. 523, 30 [1936]. sitzt und wie sich diese zu denen der idealen Lösung und denen empirischer Lösungen von FM verhalten, wie sie durch die Solvatation modifiziert werden, und ob es charakteristische Effekte gibt, welche durch die Solvatation nicht erklärt werden können. Eine Antwort hierauf kann nur durch eine vollständige exakte Theorie der athermischen Lösung auf der Grundlage der statistischen Mechanik gegeben werden. Die Schwierigkeiten bei der Aufstellung einer solchen Theorie beruhen im wesentlichen darauf, daß man bei verschiedener Größe der Moleküle die Zahl der Komplexionen nicht durch einfache Vertauschung ermitteln kann. Sie wurden zum ersten Male in einer grundlegenden Arbeit von R.H.Fowler und G.S.Rushbrooke ${ }^{34}$ überwunden für den speziellen Fall, daß die gelösten Moleküle doppelt so groß sind wie die des LM. Es ergaben sich merkliche, obgleich nicht sehr beträchtliche Abweichungen vom R a oult schen Gesetz.

Eine statistische Behandlung der Lösungen von FM wurde im Anschluß an die erwähnten Überlegungen K. H. M e y e $\mathrm{r} \mathrm{s}^{33}$ durch M. C. Hug g in s ${ }^{31}$ und P. J. F l o r $y^{35}$ gegeben. Sie erhielten eine gegenüber der idealen Lösung wesentlich erhöhte Verdünnungsentropie*.

Vom Verfasser ${ }^{1}$ wurde auf der Grundlage der Arbeit von Fowler und Rushbrooke eine Theorie der athermischen Lösung für beliebige Molekülgröße und -gestalt entwickelt und gezeigt, daß die osmotischen Eigenschaften der Lösungen hochpolymerer Substanzen aus denen der athermischen Lösung erklärt werden können. Für die Diskussion der Eigenschaften gelöster FM reicht dieser Ansatz jedoch nicht aus. Die Lösungen der hochpolymeren FM sind in den weitaus meisten Fällen polydispers, d. h. sie enthalten Moleküle der verschiedensten Größen ${ }^{36}$. Dies gilt auch für fraktionierte Produkte, wie sich mittels der Ultrazentrifuge unmittelbar zeigen läßt $\mathrm{t}^{37}$ und auch theoretisch

${ }_{30}$ P ow ell, Clark u. Ey ring, J. Chem. Physics 9, 268 [1941].

31 M. C. Hu g g ins, J. Chem. Physics 9, 440 [1941].

32 Trans. Faraday Soc. 33, 151 [1937].

33 Helv. chim. Acta 23, 1063 [1940].

34 Trans. Faraday Soc. 33, 1272 [1937].

35 J. Chem. Physics 9, 660 [1941].

* Eine Diskussion dieser Theorien und ihres Verhältnisses zu der hier entwickelten wird an anderer Stelle erfolgen.

36 H. St a u ding er, Ber. dtsch. chem. Ges. 59, 3019 [1926].

${ }_{37}$ R. Si g ner u. O. Gr o ß, Helv. chim. Acta 17, 726 [1934]. 
einleuchtet. Die Annahme, daß die Polydispersität ohne Einfluß auf die thermodynamischen Eigenschaften ist und man einfach in die für binäre Systeme entwickelten Gleichungen entsprechende Mittelwerte einsetzen kann, bedarf jedenfalls der näheren Prüfung.

\section{Statistische Theorie der poly- dispersen athermischen Lösung}

Für das thermodynamische Potential einer Lösung $F_{L}$ ergibt die Thermodynamik ${ }^{38}$ den Ausdruck

$$
F_{L}=A_{L}^{o}+V_{0} p\left(1-\frac{1}{2} x p\right) .
$$

$\left(A_{L}^{o}=\right.$ freie Energie nach Helmholtz, $V_{0}=\mathrm{Vo}_{0}$ lumen, beide für den Druck $p=0 ; x=$ Kompressibilität.) Für gewöhnliche Drucke werde $x$ von $p$ unabhängig angenommen. Ferner gilt, wenn man die von E. A. G u g g e n h e i m ${ }^{38}$ für binäre Lösungen angegebene Gleichung für eine beliebige Zahl von Komponenten verallgemeinert,

$$
\begin{aligned}
A_{L}^{o}=-k T\left[\sum_{n} N_{1 n}\left(\ln \frac{G^{\prime}}{N_{1 n}}+1\right)\right. \\
\left.+N_{2}\left(\ln \frac{G^{\prime}{ }_{2}}{N_{2}}+1\right)+\ln B(T)\right] .
\end{aligned}
$$

( $k=\mathrm{B}$ oltzm a n n sche Koǹstante, $N_{1 n}=$ Zahl der FM vom Polymerisationsgrad $n, N_{2}=$ Zahl der LM-Moleküle, $G^{\prime}=$ Verteilungsfunktionen (partition functions) der kinetischen und inneren Energie der gelösten Moleküle, $B(T)=$ Verteilungsfunktion der potentiellen Energie des ganzen Systems. $G^{\prime}$ und $B$ hängen im allgemeinen von $p$ ab und sind hier auf $p=0$ bezogen.) $B$ ist gegeben durch

$$
B(T)=\int \ldots \int \exp \left(-\frac{E}{k T}\right) d \tau .
$$

( $E=$ potentielle Energie einer Konfiguration, $d \tau$ $=$ Volumenelement des $3\left(\Sigma n N_{1 n}+N_{2}\right)$ - dimensionalen Phasenraumes.) Das Integral Gl. (9) läßt sich umformen auf Grund der folgenden Sätze:

1. Das von der Lösung eingenommene Volumen $V$ wird nicht durch das Gefäß, sondern durch die zwischenmolekularen Kräfte bedingt. Ersteres bestimmt nur die Zahl der Moleküle, die im Gleichgewicht als Dampf anwesend sind. Sie sei gegen die der flüssigen Phase zu vernachlässigen.

${ }^{33}$ E. A. Guggenheim, Proc. Roy. Soc. [London] (A) 135, 726 [1934].
2. Aus dem Wert der Verdampfungswärme (im Vergleich zu $k T$ ) und der geringen Kompressibilität folgt, daß $E$ ein scharfes Minimum besitzt für gewisse (nicht alle) Konfigurationen, bei welchen sich alle Moleküle in dem Volumen $V$ befinden. Für alle Konfigurationen, welche ein wesentlich größeres oder kleineres Volumen als $V^{\prime}$ beanspruchen, wird $E$ praktisch unendlich. Ihr Beitrag zum lntegral kann daher vernachlässigt werden. Das gleiche gilt für alle Konfigurationen, bei welchen. FM gespalten werden.

3. Das Minimum von $E$ habe die Form

$$
\text { . } E_{\min }=\frac{1}{2}\left(z^{\prime} \varepsilon_{11} \sum n N_{1 n}+z \varepsilon_{22} N_{2}\right) \text {. }
$$

$\left(\varepsilon_{11}=\right.$ Wechselwirkungsenergie der FM pro Baustein, $\varepsilon_{22}=$ Wechselwirkungsenergie der LM-Moleküle pro. Molekül, $z=$ Zahl der Nachbarn eines LM-Moleküls, $z^{\prime}=$ Zahl der einem Bausteinmolekül benachbarten LM-Moleküle.)

Damit nimmt Gl. (9) die Gestalt an

$B(T)=\exp \left(-\frac{z^{\prime} \varepsilon_{11} \sum n N_{1 n}+z \varepsilon_{22} N_{2}}{2 k T}\right) \int \ldots \int d \tau$,

wo das Integral über alle diejenigen Teile des $3\left(\Sigma n N_{1 n}+N_{2}\right)$-dimensionalen Phasenraumes zu erstrecken ist, welche mit Gl. (10) verträglich sind. Zur Berechnung dieses Integrals, welches das Übervolumen im Phasenraum darstellt, mögen noch folgende Annahmen eingeführt werden:

4. Jede in Betracht kommende Anordnung der Moleküle läßt sich darstellen durch Überlagerung einer gitterartigen „Standard-Konfiguration“ mit zwischenmolekularen Schwingungen usw. ${ }^{34}$. Die Gitterpunkte seien durch die Bausteine der FM und LM-Moleküle besetzt.

5. Für das Volumen gilt die Beziehung

$$
V=\Sigma N_{1 n}^{\prime} v_{1 n}+N_{2} v_{2},
$$

wo $v_{1 n}$ und $v_{2}$ die Partialvolumina pro Molekül sind, d.h. die Volumina, in welchen sich jenes Molekül unabhängig von den anderen und ohne Beeinträchtigung der Gl. (10) bewegen kann.

6. Es sei

$$
\Sigma n N_{1 n} \ll N_{2} \text {. }
$$

Die Sätze 3., 4. und 5. stellen die statistische Definition der athermischen Lösung dar.

Auf Grund von 4. kann man das Integral der Gl. (11) in zwei Anteile zerlegen, indem man ein- 
mal über die molekularen Partialvolumina und dann über sämtliche Standard-Konfigurationen integriert. Der erste Teil liefert das Produkt

$$
\prod_{n} v_{1 n}^{o^{N_{1}}{ }^{n}} \cdot v_{2}^{O^{N_{2}}}
$$

Die Berechnung des zweiten, des sog. „Kombinationsfaktors " ${ }^{34}$ ist gleichbedeutend mit der $\mathrm{Ab}$ zählung der Konfigurationen, in welchen $\Sigma N_{1 n}$ FM und $N_{2}$ LM-Moleküle $\Sigma n N_{1 n}+N_{2}$ Gitterpunkte besetzen können. Sie stellt den Kern des Problems dar.

Da die FM und die Moleküle des LM nicht gegeneinander vertauscht werden können, muß man so vorgehen, daß man die Bausteine der FM gegen die LM-Moleküle vertauscht unter Einhaltung der „Kohärenzbedingung" ${ }^{11}$, daß bei jeder Vertauschung die gleichen $n$ Bausteine zu einem FM vom Polymerisationsgrad $n$ verbunden bleiben. Es sei zunächst nur ein FM vom Polymerisationsgrad $n$ in der Lösung vorhanden. Dann können infolge der Kohärenzbedingung die $n$ Bausteinmoleküle nur gleichzeitig und nur mit solchen LM-Molekülen vertauscht werden, welche in ihrer momentanen Anordnung ebenfalls eine mögliche Konfiguration des FM darstellen. Die Zahl derartiger Anordnungen im Gitter der Lösung sei $\Lambda_{n}+1 . \Lambda_{n}$ werde als Zahl der ,virtuellen FM“ vom Polymerisationsgrad $n$ bezeichnet ${ }^{1}$; sie ist gleich der Zahl der möglichen Anordnungen eines FM im Gitter der Lösung, vermindert um die durch reelle FM besetzten. Kommt noch ein zweites FM der gleichen Größe hinzu, so können die Bausteine der beiden auch untereinander vertauscht werden; wegen der Kohärenzbedingung ergibt dies jedoch nur eine neue Vertauschung. Die Zahl der Anordnungen wird daher $\left(\Lambda_{n}+1\right) \quad\left(\Lambda_{n}+2\right)$ und für $N_{1 n}$ FM $\left(\Lambda_{n}+N_{1 n}\right) ! / \Lambda_{n}$ !. Entsprechendes gilt für die FM jeder anderen Größe. Man kann ferner alle kleineren FM durch LM-Moleküle zu FM der maximalen Größe ergänzen und dann die FM verschiedener Größe unter Beteiligung der Ergänzung vertauschen. Dies ergibt weitere $\left(\Sigma N_{1 n}\right) ! / \Pi N_{1 n}$ ! Anordnungen. Aus der Voraussetzung 6. und dem unten über die Größen $\Lambda_{n}$ Ausgeführten ergibt sich, daß diese Anordnungen unbedenklich gegen die obigen vernachlässigt werden können. Schließlich kommen noch weitere $N_{2}$ !-Anordnungen durch Vertauschung der Moleküle des LM untereinander hinzu. Man erhält somit

$$
B(T)=\exp \left(-\frac{z^{\prime} \varepsilon_{11} \sum n N_{1 n}+z \varepsilon_{22} N_{2}}{2 k T}\right) \prod_{n}\left[\frac{\left(N_{1 n}+\Lambda_{n}\right) !}{\Lambda_{n} !} v_{1 n}^{o}{ }^{N_{1 n}}\right] N_{2} ! v_{2}^{o}{ }^{N 2}
$$

Mit Benutzung der Stirlingschen Näherungsformel folgt aus Gl. (8) und (13)

$A_{L}^{o}=-k T\left[\sum_{n} N_{1 n}\left(\ln \frac{G_{1 n}^{\prime} v_{1 n}^{o}\left(N_{1 n}+\Lambda_{n}\right)}{N_{1 n}}-1 / 2 \frac{z^{\prime} \varepsilon_{11} n}{k T}\right)\right.$

Setzt $\operatorname{man}^{32}$

$$
\left.+\sum_{n} \Lambda_{n}\left(\ln \frac{\left(G^{\prime}{ }_{2} v_{2}^{O}\right) \frac{N_{2}}{\Lambda_{n}}\left(N_{1 n}+\Delta_{n}\right)}{\Lambda_{n}}-1 / 2 \frac{z \varepsilon_{22} N_{2}}{k T \Lambda_{n}}\right)\right] \text {. }
$$

$$
\varkappa=\frac{\sum \varkappa_{1 n} N_{1 n} v_{1 n}^{o}+\varkappa_{2} N_{2} v_{2}^{o}}{\sum N_{1 n} v_{1 n}^{o}+N_{2} v_{2}^{o}}
$$

so erhält man aus Gl. (7), (12), (14) und (15) für das thermodynamische Potential

$$
\begin{aligned}
F_{L} & =k T\left[\sum_{n} N_{1 n} \ln \frac{N_{1 n}}{N_{1 n}+\Lambda_{n}}+\sum_{n} \Lambda_{n} \ln \frac{\Lambda_{n}}{N_{1 n}+\Lambda_{n}}+1 / 2-\frac{z^{\prime} \varepsilon_{11} \sum_{n} n N_{1 n}+z \varepsilon_{22} N_{2}}{k T}\right. \\
& \left.-\sum N_{1 n} \ln G_{1 n}-N_{2} \ln G_{2}+\frac{p}{k T} \sum_{n} N_{1 n} v_{1 n}^{o}\left(1-1 / 2 \varkappa_{1 n} p\right)+\frac{p}{k T} N_{2} v_{2}^{o}\left(1-1 / 2 \varkappa_{2} \mathrm{p}\right)\right],
\end{aligned}
$$

wo $G=G^{\prime} v_{0}$ die Verteilungsfunktionen der gesamten inner- und zwischenmolekularen Schwingungen usw. sind.

Die Zahl der virtuellen FM in einem Gitter läßt sich allgemein so ermitteln, daß man, von einem
Gitterpunkt ausgehend, nach festgelegterVorschrift das FM aufbaut und die Zahl der sich hierbei ergebenden Möglichkeiten für jeden Gitterpunkt bestimmt $^{1}$. Für ein unbegrenztes Gitter aus LM-Molekülen ist offenbar einfach $\Lambda_{0 n} \sim N_{2}$, weil jeder 
Gitterpunkt die gleiche Zahl von Möglichkeiten für den Aufbau des virtuellen FM bietet. Es wird somit

$$
\Lambda_{0 n}=N_{2} f(z)_{n} \text {. }
$$

Die Funktion $f(\boldsymbol{z})_{n}$ hängt jedoch von den Eigenschaften ab, welche dem FM zugeschrieben werden. Für starre FM, wie sie insbesondere nach Staudingers ${ }^{39}$ Auffassung in den Lösungen vorliegen, ist sie einfach gleich der Zahl der direkt benachbarten Gitterpunkte $z$. Es gilt hier also

$$
\Lambda_{0 n}=N_{2} z \text {. }
$$

Für bewegliche FM, wie sie von W. Kuhn ${ }^{40}$, Haller ${ }^{21}$, E. Guth u. H. M a r k ${ }^{41}$, K.H.Meyer ${ }^{7,33}$ angenommen werden, wird $\Lambda_{0 n}$ eine Funktion des Polymerisationsgrades. Der Fall, daß der dritte und jeder weitere Baustein $z-1$ Gitterpunkte besetzen kann, werde als ideale Beweglichkeit be-

$$
\Lambda_{n}=\Lambda_{n}\left(N_{11}, N_{12}, \ldots N_{1 n}, N_{2}\right) ; \frac{\partial \Lambda_{n}}{\partial N_{11}}<
$$

$\Lambda_{n}$ muß offenbar gleich sein der Zahl der im Gitter der Lösung vorhandenen virtuellen $\mathrm{FM} \Lambda_{0 n}$, vermindert um die reellen FM und die durch den ,Störungseffekt" ausfallenden virtuellen FM. Die letzteren kann man in erster Näherung (d. h. wenn die Anordnungen, bei welchen einzelne FM benachbart sind, unberücksichtigt bleiben, was durch die Voraussetzung 6. gerechtfertigt wird) proportional $\Sigma N_{1 n}$ setzen. Man erhält somit

$\Lambda_{n}=\left(N_{2}+\sum_{n^{\prime}} n^{\prime} N_{1 n^{\prime}}\right) f(z)_{n}-\sum_{n^{\prime}}\left(a_{n n^{\prime}}+1\right) N_{1 n^{\prime}}$.

$a_{n n^{\prime}}$ ist die Zahl der virtuellen FM vom Polymerisationsgrad $n$, welche infolge der Anwesenheit eines reellen FM vom Polymerisationsgrad $n^{\prime}$ ausfallen. Es ist gleich der Summe der Produkte aus der Zahl der von einem Gitterpunkt aus aufzubauenden virtuellen FM und dem Bruchteil, der davon ausfällt, über alle dem reellen FM benachbarten Gitterpunkte innerhalb der ,Störungssphäre". Für starre FM hat man daher

$$
a_{n n^{\prime}}=n^{\prime} z a_{n},
$$$$
a_{n}=(z-2)\left[\frac{1}{z}+\frac{z-1}{z^{2}}+\frac{(z-1)^{2}}{z^{3}}+\ldots \frac{(z-1)^{n-1}}{z^{n}}\right] .
$$

zeichnet (vergl. die Abbildgn. bei K. H. M e y e ${ }^{33}$ ). Hier ist

$$
\Lambda_{0 n}=N_{2} z(z-1)^{n-2} \approx N_{2}(z-1)^{n} .
$$

In Wirklichkeit ist das Gitter der Lösung durch die Gefäßwände begrenzt, und für die an der Begrenzung liegenden Gitterpunkte ist die Zahl der Möglichkeiten zum Aufbau virtueller FM kleiner als im Inneren der Lösung. Diese Gitterpunkte können indessen gegen die Gesamtzahl vernachlässigt werden ${ }^{34}$. Von entscheidender Bedeutung ist aber die Tatsache, daß auch die den reellen FM benachbarten Gitterpunkte an einer Begrenzung liegen und somit eine geringere Zahl ,freier" Nachbarn besitzen als die im Inneren des reinen LM befindlichen ${ }^{1,34}$ (,Störungseffekt"). Es muß daher gelten

$<0, \frac{\partial \Lambda_{n}}{\partial N_{12}}<0, \ldots \frac{\partial \Lambda_{n}}{\partial N_{1 n}}<0$.

für ideal bewegliche

$$
a_{n n^{\prime}}=n^{\prime}(z-1)^{n} a_{n} .
$$

Der „Störungsparameter" $\alpha_{n}$ ist die Summe der ausfallenden Bruchteile über die Zahl der benachbarten gestörten Gitterpunkte pro Baustein des reellen FM. Eine exakte Berechnung von $\alpha_{n}$ ist noch nicht möglich, weil darin die Struktur der flüssigen Phase und die genaue Beweglichkeit der FM eingeht. Umgekehrt sind aus der Diskussion der experimentellen $\alpha_{n}$-Werte Aufschlüsse über diese Fragen $\mathrm{zu}$ erwarten. Allgemein muß $\alpha_{n}$ um so größer sein, je geringer die Beweglichkeit ist, weil der Bruchteil der ausfallenden virtuellen FM wächst. Für starre FM gilt näherungsweise

$$
a_{n}=\frac{z-2}{z} n,
$$

was für $z=4$ und $n=1000 \alpha_{n}=500$ ergibt. Für ideal bewegliche FM hat man

Die Beweglichkeit der realen FM dürfte zwischen den beiden Grenzfällen liegen (vergl. dazu ${ }^{33}$ und die Modellversuche von H.A.Stua $\mathrm{rt}^{42}$ ) und von den speziellen Eigenschaften des Systems abhängen. Da die Beweglichkeit mit der Temperatur

42 Naturwiss. 31, 123 [1943].

\footnotetext{
${ }^{39} \mathrm{H}$. St a u dinger, Organische Kolloidchemie, Braunschweig 1941.

40 Kolloid-Z. 68, 2 [1934].

${ }^{41}$ Mh. Chem. 65, 93 [1935].
}

Danach muß $\alpha_{n}$ für höhere Polymerisationsgrade praktisch unabhängig von $n$ sein. Für das obige Zahlenbeispiel erhält man aus Gl. (21b) $\alpha_{n}=2$. 
wächst, ist anzunehmen, daß $\alpha_{n}$ auch eine Funktion von $T$ ist. Dieser Einfluß möge indessen zunächst vernachlässigt werden.

Für die weitere Rechnung ist es zweckmäßig, die auf das Molekül bezogenen Größen $N, \Lambda, v, \varepsilon$, $k$ durch die auf das Mol bezogenen $n, \lambda, V, E, R$ zu ersetzen und die Abkürzung

$$
\gamma_{n}=\frac{n_{1 n}}{n_{1 n}+\lambda_{n}}
$$

einzuführen. Sie werde als virtueller Molenbruch bezeichnet.
III. Die chemischen Potentiale; die Verdünnungsentropie; die Aktivität des LM

Die Gl. (16) enthält implicite alle thermodynamischen Eigenschaften der polydispersen athermischen Lösung. Es mögen nun einige Funktionen daraus abgeleitet werden, die teils für weitere Berechnungen, teils zum Vergleich mit dem Experiment geeignet sind.

Die Gibbsschen chemischen Potentiale sind definiert durch die Gleichung

$$
\mu_{i}=\left(\frac{\partial F_{L}}{\partial n_{i}}\right)_{T, p}
$$

Man erhält demgemäß aus Gl. (16) und (22)

$$
\begin{aligned}
\mu_{1 n}=R T\left[\ln \gamma_{n}+\frac{\partial \lambda_{n}}{\partial n_{1 n}} \ln \left(1-\gamma_{n}\right)+\sum_{n^{\prime}}\right. & \frac{\partial \lambda_{n^{\prime}}}{\partial n_{1 n}} \ln \left(1-\gamma_{n^{\prime}}\right) \\
& \left.+1 / 2 \frac{z^{\prime} n E_{11}^{\prime}}{R T}-\ln G_{1 n}+\frac{p V_{1 n}^{o}}{R T}\left(1-\frac{1}{2} \varkappa_{1 n} p\right)\right]
\end{aligned}
$$

wo die Summe $\Sigma_{n}^{\prime}$ über alle Werte von $n$, mit Ausnahme dessen, zu dem das Potential gehört, zu erstrecken ist. Ferner ist

$$
\mu_{2}=R T\left[\sum_{n} \frac{\partial \lambda_{n}}{\partial n_{2}} \ln \left(1-\gamma_{n}\right)+1 / 2 \frac{z E_{22}}{R T}-\ln G_{2}+\frac{p V_{2}^{o}}{R T}\left(1-\frac{1}{2} \varkappa_{2} p\right)\right] .
$$

Für die Verdünnungsentropie folgt aus Gl. (6) und (25)

$$
s_{2}-s_{02}=-R \sum_{n} \frac{\partial \lambda_{n}}{\partial n_{2}} \ln \left(1-\gamma_{n}\right) .
$$

Auf Grund der Voraussetzung 6. in II. kann man die Entwicklung des Logarithmus mit dem linearen Gliede abbrechen. Vernachlässigt man aus dem gleichen Grunde im Nenner von (22) $n_{1 n}$ gegen $\lambda_{n}$ und $\Sigma n N_{1 n}$ gegen $N_{2}$, ferner wegen Gl. (20a) bzw. (20 b) 1 gegen $a_{n n^{\prime}}$, so erhält man mit Gl. (19)

$$
s_{2}-s_{02}=R \sum_{n} \frac{f(z)_{n} n_{1 n}}{n_{2} f(z)_{n}-\sum_{n^{\prime}} a_{n n^{\prime}} n_{1 n^{\prime}}} .
$$

Führt man die auf das reine LM bezogene Molkonzentration $\mathrm{c}_{n}=n_{1 \mathrm{n}} / n_{2} V_{02}$ ein, so wird

$$
s_{2}-s_{02}=R V_{02} \sum_{n} \frac{c_{n}}{1-\frac{V_{02}}{f(z)_{n}} \sum_{n^{\prime}} a_{n n^{\prime}} c_{n^{\prime}}} \text {. }
$$

Zur weiteren Auswertung dieser Gleichung benötigt man die Verteilungsfunktion der Polymerisationsgrade. Sie werde definiert durch die Gleichung

$$
c_{n}=c f(n)
$$

wo $c$ die gesamte molare Konzentration der gelösten FM, bezogen auf das Volumen des reinen LM, bezeichnet. Sie hängt mit der von G. V. $\mathrm{Sch} \mathrm{u} \mathrm{l} \mathrm{z}{ }^{43,44}$ eingeführten ,Häufigkeitsverteilungsfunktion" $h(n)$ zusammen durch die Beziehung

$$
f(n)=\frac{h(n)}{\sum h(n)} .
$$

Es gilt ferner

$$
\sum_{n=0}^{n=\infty} f(n)=1
$$

Führt man die Gewichtskonzentrationen $\boldsymbol{c}_{\boldsymbol{n}} \boldsymbol{g}$ $=M_{B} n c_{n}$ und $c_{g}=M_{B} c \sum n f(n)$ ein (wo $M_{B}$ das Molekulargewicht des Bausteinmoleküls ist), so geht Gl. (29) über in

$$
\begin{aligned}
& s_{2}-s_{02}=R V_{02} \\
& \sum_{n} \frac{f(n) c_{g}}{M_{B} \sum_{n} n f(n)\left[1-\frac{V_{02} \sum_{n^{\prime}} a_{n n^{\prime}} f\left(n^{\prime}\right)}{M_{B} \sum n f(n) f(z)_{n}} c_{g}\right] .}
\end{aligned}
$$

${ }_{43}$ Schulz, Z. physik. Chem. (B) 32, 27 [1936].

$44 \mathrm{Schulz}$, Z. physik. Chem. (B) 47, 155 [1940]. 
Entwickelt man den Nenner und bricht mit dem zweiten Gliede ab, so erhält man wegen Gl. (31)

$$
\begin{aligned}
& s_{2}-s_{02}=\frac{R V_{02}}{M_{B} \sum n f(n)} c_{g} \\
& +\frac{R V_{02}{ }^{2}}{\left[M_{B} \sum_{n} n f^{\prime}(n)\right]^{2}} \sum_{n} \frac{\sum_{n^{\prime}} a_{n n^{\prime}} f\left(n^{\prime}\right)}{f(z)_{n}} f(n) c_{g}{ }^{2} .
\end{aligned}
$$

Der Ausdruck $M_{B} \sum n f(n)$ stellt das osmotisch bestimmte mittlere Molekulargewicht $\bar{M}_{1}$ dar, wie in einer späteren Untersuchung gezeigt wird, $\Sigma n f(n)$ den mittleren Polymerisationsgrad $\bar{n}$. Daraus folgt schließlich bei Berücksichtigung der Gl. (17a), (20a) und (31)

$$
s_{2}-s_{02}=\frac{R V_{02}}{\bar{M}_{1}} c_{g}+\frac{R V_{02}{ }^{2} \bar{\alpha}_{n}}{M_{B} \bar{M}_{1}} c_{g}{ }^{2},
$$

wo $\bar{\alpha}_{n}$ der mit der. Verteilungsfunktion gebildete Mittelwert von $\alpha_{n}$ ist. Diese Gleichung möge nun mit einigen thermodynamischen Messungen an Lösungen hochpolymerer FM verglichen werden.

\begin{tabular}{|c|c|c|c|c|c|c|}
\hline$w$ & $\begin{array}{c}\bar{N}_{1} \\
\cdot 10^{5}\end{array}$ & $\begin{array}{c}c_{g} \\
\mathrm{~g} / \mathrm{l}\end{array}$ & $\begin{array}{c}\left(H_{2}-H_{02}\right) \\
\text { exp } \\
\text { cal }\end{array}$ & $\begin{array}{c}\left(s_{2}-s_{02}\right) \\
\text { id } \\
\text { cal/grad } \\
\cdot 10^{5}\end{array}$ & $\begin{array}{c}\left(s_{2}-s_{02}\right) \\
\text { exp } \\
\text { cal grad } \\
\cdot 10^{5}\end{array}$ & $\begin{array}{c}\left(s_{2}-s_{02}\right. \\
\text { theor } \\
\text { cal/grad } \\
\cdot 10^{5}\end{array}$ \\
\hline 0,0348 & 1,23 & 30,81 & 0,02 & 2,441 & 36,28 & 36,28 \\
0,0466 & 1,67 & 41,78 & 0,045 & 3,298 & 62,66 & 65,50 \\
\hline
\end{tabular}

Tab. 1. Verdünnungsentropie des Systems KautschukToluol. Messungen von K. H. Meyer, E. Wolff und Ch. B o is s o nnas ${ }^{15}$.

$\bar{M}_{1}=270000 \pm 30000 ; M_{2}=92,14 ; M_{B}=68,12 ;$

$T=303,2^{\circ}$ abs. $; d_{2}=0,86 \mathrm{~g} / \mathrm{ccm} ; \bar{a}_{n}=2,96 \cdot 10^{2}$.

\begin{tabular}{|c|c|c|c|c|c|c|}
\hline$w$ & $\begin{array}{c}\bar{N}_{1} \\
10^{5}\end{array}$ & $\begin{array}{c}c_{g} \\
\mathrm{~g} / \mathrm{l}\end{array}$ & $\begin{array}{c}\left(H_{2}-H_{02}\right. \\
\text { exp } \\
\mathrm{cal}\end{array}$ & $\begin{array}{c}\left(s_{2}-s_{02}\right) \\
\text { id } \\
\text { cal/grad } \\
\cdot 10^{5}\end{array}$ & $\begin{array}{c}\left(s_{2}-s_{02}\right) \\
\text { exp } \\
\text { cal/grad } \\
\cdot 10^{5}\end{array}$ & $\begin{array}{c}\left(s_{2}-s_{02}\right) \\
\text { theor } \\
\text { cal/grad } \\
\cdot 10^{5}\end{array}$ \\
\hline 0,0285 & 9,1 & 25,07 & $\begin{array}{c}0,00 \\
\pm \\
0,01\end{array}$ & 18,14 & 36,28 & 36,28 \\
0,0405 & 13,0 & 36,08 & $\begin{array}{c} \pm, 01 \\
\pm \\
0,01\end{array}$ & 25,73 & 62,66 & 63,29 \\
\hline
\end{tabular}

Tab. 2. Verdünnungsentropie des Systems GuttaperchaToluol. Messungen von E. W olf $\mathrm{f}^{\mathbf{1 6}}$.

$\bar{M}_{1}=300000 \pm 2000 ; M_{2}=92,14 ; M_{B}=68,12 ;$

$T=303,2^{\circ}$ abs. $; d_{2}=0,86 \mathrm{~g} / \mathrm{ccm} ; \bar{\alpha}_{n}=0,266 \cdot 10^{2}$.

\begin{tabular}{|c|r|c|c|c|c|c|}
\hline $\boldsymbol{w}$ & $\begin{array}{c}\bar{N}_{1} \\
\cdot 10^{5}\end{array}$ & $\begin{array}{c}c_{g} \\
\mathrm{~g} / 1\end{array}$ & $\begin{array}{c}\left(H_{2}-H_{02}\right) \\
\text { exp } \\
\text { cal }\end{array}$ & $\begin{array}{c}\left(s_{2}-s_{02}\right) \\
\text { id } \\
\text { cal/grad } \\
\cdot 10^{5}\end{array}$ & $\begin{array}{c}\left(s_{2}-s_{02}\right. \\
\text { exp } \\
\text { cal/grad } \\
\cdot 10^{5}\end{array}$ & $\begin{array}{c}\left(s_{2}-s_{02}\right) \\
\text { theor } \\
\text { cal/grad } \\
\cdot 10^{5}\end{array}$ \\
\hline 0,01705 & 2,6 & 16,27 & 0,015 & 4,664 & 8,397 & 8,397 \\
0,0333 & 5,9 & 32,31 & 0,09 & 10,747 & 13,18 & 25,487 \\
0,0612 & 10,7 & 61,14 & 0,17 & 19,792 & 69,20 & 72,522 \\
\hline
\end{tabular}

Tab. 3. Vérdünnungsentropie des Systems NitrocelluloseCyclohexanon. Messungen von Ch. Boissonnas und K. H. M e y e ${ }^{13}$.

$$
\begin{aligned}
& \bar{M}_{1}=70000 ; M_{2}=98,1 ; M_{B}=280 ; \\
& T=303,5^{\circ} \text { abs. } \bar{a}_{n}=1,27 \cdot 10^{2} .
\end{aligned}
$$

\begin{tabular}{|c|c|c|c|c|}
\hline$w$ & $\bar{N}_{1} \cdot 10^{5}$ & 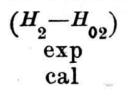 & $\mid \begin{array}{c}\left(s_{2}-s_{\text {o2 }}\right) \text { id } \\
\text { cal } / \text { grad } \\
\cdot 10^{5}\end{array}$ & $\mid \begin{array}{c}\left(s_{2}-s_{09}\right) \exp \\
\text { cal } / \operatorname{grad}^{5} \\
-10^{5}\end{array}$ \\
\hline 0,0043 & 1,73 & $-0,006$ & 3,299 & 2,639 \\
\hline 0,0092 & 3,68 & $-0,015$ & 7,258 & 7,588 \\
\hline 0,0225 & 9,03 & $-0,155$ & 17,82 & $-0,9898$ \\
\hline 0,0438 & 18,29 & $-0,440$ & 36,29 & $-12,21$ \\
\hline
\end{tabular}

\begin{tabular}{|c|r|l|l|l|l|}
\hline System & $\bar{M}_{1}$ & $\begin{array}{c}\bar{\alpha}_{n \text { exp }} \\
\cdot 10^{-2}\end{array}$ & $z$ & $\begin{array}{l}\bar{\alpha}_{n \text { theor }} \\
\cdot 10^{-2}\end{array}$ & $\begin{array}{l}\text { Lite- } \\
\text { ratur }\end{array}$ \\
\hline Kautschuk-Benzin & 280000 & 3,289 & $\mathbf{2 , 1 7 4}$ & 3,289 & $(17)$ \\
Kautschuk-Benzin & 150000 & 1,703 & 2,168 & 1,762 & $(17)$ \\
Kautschuk-Benzin & 85000 & 0,949 & 2,165 & 0,998 & $(17)$ \\
Kautschuk-Benzin & 38000 & 0,332 & 2,126 & 0,446 & $(17)$ \\
Kautschuk-Toluol & 270000 & 2,96 & 2,161 & 2,96 & $(15)$ \\
Guttapercha-Toluol & 30000 & 0,266 & 2,129 & 0,329 & $(16)$ \\
\hline
\end{tabular}

Tab. 4. Störungsparameter und Zahl der Gitternachbarn.

Tab.5. Verdünnungsentropie des Systems Acetylcellulose-Tetrachloräthan. Messungen von $\mathrm{O}$. $\mathrm{H}$ a g g e $\mathrm{r}$ und A. J. A. van der $\mathrm{Wy} \mathrm{k}^{14}$.

$$
\begin{aligned}
& \bar{M}_{1}=41800 \pm 2000 ; M_{2}=167,9 ; M_{B}=288 ; \\
& T=303,1^{\circ} \mathrm{abs} .
\end{aligned}
$$

\begin{tabular}{|c|c|c|c|}
\hline $\begin{array}{c}c_{\mathrm{g}} \\
\mathrm{g} / \mathrm{J}\end{array}$ & $\begin{array}{c}\left(H_{2}-H_{02}\right) \exp \\
\mathrm{cal}\end{array}$ & $\begin{array}{c}\left(s_{2}-s_{02}\right) \text { id } \\
\text { cal/grad } \cdot 10^{5}\end{array}$ & $\begin{array}{c}\left(s_{2}-s_{02}\right) \exp \\
\text { cal/grad } \cdot 10^{5}\end{array}$ \\
\hline 10,0 & 0,0045 & 1,798 & 2,098 \\
19,8 & 0,0206 & 3,596 & 3,363 \\
29,5 & 0,0393 & 5,395 & 5,061 \\
\hline
\end{tabular}

Tab. 6. Verdünnungsentropie des Systems Nitrocellulose-Aceton. Messungen von G. V. Sch u l z ${ }^{12}$.

$\bar{M}_{1}=82000 ; M_{2}=58,1 ; M_{B}=280 ; T=300,2^{\circ}$ abs. 


\begin{tabular}{|c|c|c|c|}
\hline $\begin{array}{c}c_{\mathrm{g}} \\
\mathrm{g} / 1\end{array}$ & $\begin{array}{c}\left(\mathrm{H}_{2}-\mathrm{H}_{02}\right) \exp \\
\mathrm{cal}\end{array}$ & $\begin{array}{c}\left(s_{2}-s_{02}\right) \mathrm{id} \\
\mathrm{cal} / \mathrm{grad} \cdot 10^{5}\end{array}$ & $\begin{array}{c}\left(s_{2}-s_{02}\right) \exp \\
\mathrm{cal} / \mathrm{grad} \cdot 10^{5}\end{array}$ \\
\hline 9,9 & 0,0047 & 2,650 & 2,396 \\
19,6 & 0,0166 & 4,695 & 4,862 \\
29,0 & 0,0344 & 6,993 & 7,259 \\
\hline
\end{tabular}

Tab. 7. Verdünnungsentropie des Systems PolystyrolToluol. Messungen von G. V.Schulz ${ }^{12}$.

$\bar{M}_{1}=90000 ; M_{2}=92,14 ; M_{B}=101,15 ; T=300,2^{\circ} \mathrm{abs}$.

$\mathrm{Zu}$ den Tabellen $1-3$ ist folgendes zu bemerken: $\boldsymbol{d}_{\mathbf{2}}$ bedeutet die Dichte des LM in der Lösung. Die erste Spalte enthält die gewichtsmäßige Zusammensetzung in g pro $g$ Lösung, die zweite den daraus nach der Formel

$$
\bar{N}_{1}=\frac{w / \bar{M}_{1}}{(1-w) / M_{2}+w / \bar{M}_{1}}
$$

berechneten mittleren Molenbruch, Spalte 3 die nach der Formel

$$
c_{g}=\frac{w}{(1-w) / d_{2}}
$$

berechnete Gewichtskonzentration, Spalte 4 die aus der Temperaturabhängigkeit des osmotischen Druckes von den Autoren berechnete Verdünnungswärme, Spalte 5 die aus dem mittleren Molenbruch von den Autoren berechnete Verdünnungsentropie der idealen Lösung*, Spalte 6 die aus der Temperaturabhängigkeit des osmotischen Druckes von den Autoren berechnete Verdünnungsentropie*. Der erste Wert der Spalte 7 wurde dem experimentellen Wert der Spalte 6 gleichgesetzt und zur Berechnung dès Störungsparameters $\alpha_{n}$ nach Gl. (34) benutzt, die weiteren mit diesem Wert aus Gl. (34) berechnet.

Die Tabellen lassen erkennen, daß die Konzentrationsabhängigkeit der Verdünnungsentropie durch Gl. (34) befriedigend wiedergegeben wird. Auch Gee und Trelo a $\mathrm{r}^{17}$ haben gezeígt, daß die von ihnen ermittelte Verdünnungsentropie des Systems Kautschuk-Benzin durch eine quadratische Funktion der Gewichtskonzentration sich gut darstellen läßt (l. c. Abb. 13).

Die absolute Größe der anomalen Entropieeffekte wird durch den Störungsparameter $\overline{\alpha_{n}}$ bestimmt. In Tab. 4 sind die aus den Messungen von G e e und Trel o a r ${ }^{17}$ berechneten Werte für $\bar{\alpha}_{n}$ sowie die der

* Die betr. Werte der Tab. 3 wurden vom Verf. aus den Daten der Arbeit berechnet.
Tabellen 1 und 2 zusammengestellt. Spalte 4 der Tab. 4 enthält die nach Gl. ( 21 a) berechnete mittlere Zahl der nächsten Nachbarn $z$, Spalte 5 die mit dem jeweils ersten dieser Werte nach Gl. (21 a) berechneten $\overline{\alpha_{n}}$. Allgemein ergibt sich, daß die experimentellen Werte für $\bar{\alpha}_{n}$ innerhalb der nach der Theorie zu erwartenden Größenordnungen liegen. Aus Gl. (21 a), erhält man einen plausiblen und praktisch konstanten Wert für die mittlere Zahl der nächsten Gitternachbarn $\boldsymbol{z}$. Diese Gleichung stellt auch die Abhängigkeit der Größe $\overline{\alpha_{n}}$ vom Polymerisationsgrad in guter Näherung dar. Dagegen führt Gl. (21b) auf unmögliche Werte für $z$ und ergibt praktisch keine Abhängigkeit der Größe $\bar{\alpha}_{n}$ von $n$. Man muß daher annehmen, daß die FM der „Polyprene“ nur eine verhältnismäßig geringe Beweglichkeit in der Lösung besitzen.

Nach diesen Ergebnissen erscheint der Schluß gerechtfertigt, daß in erster Linie Größe, Gestalt und Beweglichkeit gelöster FM ihre charakteristischen thermodynamischen Eigenschaften bestimmen.

Aus der Theorie folgt, daß die Verdünnungsentropie unabhängig ist nicht nur von der speziellen Verteilungsfunktion, sondern von der Polydispersität als solcher; Gl. (34) unterscheidet sich von der entsprechenden, aus der Theorie der binären athermischen Lösung folgenden nur dadurch, daß Mittelwerte auftreten. Für unendliche Verdünnung erhält man als Grenzwert

$$
\lim _{c_{g}=0} \frac{\left(s_{2}-s_{02}\right)}{c_{g}}=\frac{R V_{02}}{\bar{M}_{1}},
$$

die für die ideale Lösung über den ganzen Konzentrationsbereich gültige Gleichung.

In Tab. 5-7 sind noch einige weitere Messungen zusammengestellt, bei welchen die empirische Verdünnungsentropie die aus der Theorie der idealen Lösung berechnete nicht überschreitet. Im Falle der Tab. 5 wird dies von den Autoren selbst durch die Solvatation erklärt. G.V.Schulz hatte ursprünglich $^{\ominus}$ aus seinen Messungen wesentlich höhere Entropiewerte errechnet. Sowohl die Meßmethode wie die Auswertung wurden dann eingehend von Ch. G. B o is s onnas und K. H. Meyer ${ }^{45,46}$ kritisiert. Ohne auf diese Diskussion (vergl. auch ${ }^{47}$ ) hier einzugehen, sei bemerkt, daß

45 Z. physik. Chem. (B) 40, 108 [1938].

46 Z. physik. Chem. (B) 44, 392 [1939].

${ }^{47}$ G. V. Schulz, Z. physik. Chem. (B) 45, 110 [1940]. 
die Schulzschen Ergebnisse an dem System Polystyrol-Toluol (Tab. 7) sich nur schwer in Einklang bringen lassen mit den Folgerungen, zu denen anderweitige Untersuchungen dieses $\mathrm{Sy}$ stems führen (s. o. II 2).

Für die Aktivität des LM gilt, wenn das reine LM bei Atmosphärendruck als Normalzustand gewählt wird, nach Gl. (4) und (25)

$$
a_{2}=\prod_{n}\left(1-\gamma_{n}\right)^{\frac{\partial \lambda_{n}}{\partial n_{2}}} \text {. }
$$

Durch eine der obigen analoge Rechnung erhält man

$$
\ln a_{2}=-\left(\frac{V_{02}}{\bar{M}_{1}} c_{g}+\frac{V_{02} \bar{a}_{n}}{M_{B} \bar{M}_{1}} c_{g}^{2}\right) .
$$

Aus dieser Gleichung folgt sofort die Beziehung(5). Aus Gl. (36) erhält man weiter

$$
\lim _{N_{2}=1} \frac{a_{2}}{N_{2}}=1
$$

wie es die thermodynamische Definition der Aktivität verlangt. Da die Aktivität als das Verhältnis der Flüchtigkeiten in dem betrachteten und dem Normalzustand, $f / f_{0}$, definiert ist ${ }^{2}$, folgt aus Gl. (38), daß bei der athermischen Lösung das $R$ a oul tsche Gesetz

$$
f / f_{0}=N_{2}
$$

als Grenzgesetz für unendliche Verdünnung gilt.

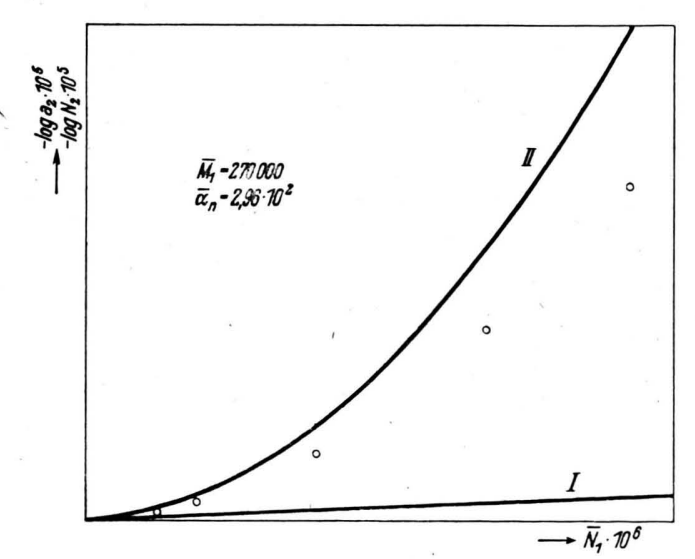

Abb. 1. Aktivität des LM im System Kautschuk-Toluol. (Messungen von K. H. Meyer, Wolff u. B o is s onna s ${ }^{15}$.) Kurve I: Ideale Lösung. Kurve II: Athermische Lösung [Gl. (37)]. Kreise: Experimentelle Werte.
In Abb. 1-3 sind die Funktionen - $\log a_{2}$ und $-\log N_{2}$ für einige Systeme dargestellt. Die Abweichung von der idealen Lösung ist deutlich erkennbar. Die Meßpunkte schmiegen sich den aus der vorstehenden Theorie [Gl.(37)] berechneten Kurven an.

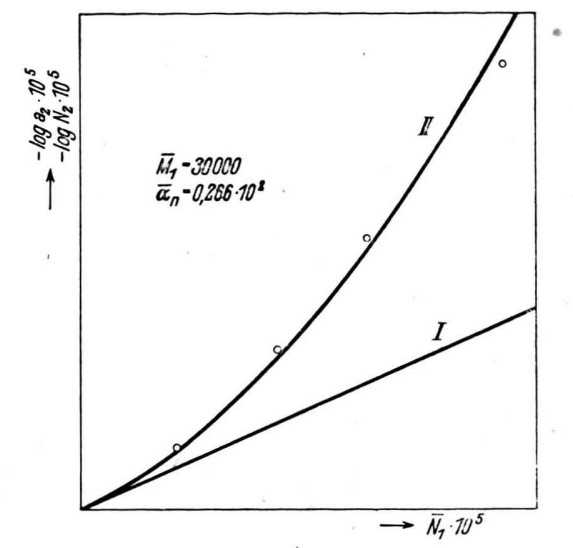

Abb. 2. Aktivität des LM im System Guttapercha-Toluol. (Messungen von E. Wolf f ${ }^{16}$.) Kurve I: Ideale Lösung.

Kurve II: Athermische Lösung [Gl. (37)].

Kreise: Experimentelle Werte.

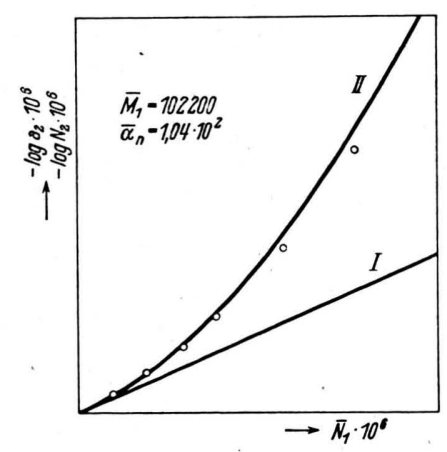

Abb. 3. Aktivität des LM im System Guttapercha-Toluol. (Messungen von $\mathrm{Staudinger}$ und $\mathrm{F}$ is ch e ${ }^{48}$.) Kurve I: Ideale Lösung. Kurve II: Athermische Lösung [Gl. (37) ]. Kreise: Experimentelle Werte.

Aus den Abb. 1-3 wie auch aus Gl. (35) und (38) ergibt sich, daß die Gesetze der athermischen Lösung bei unendlicher Verdünnung in die der idealen Lösung übergehen. Man kann diese letztere jedoch nicht zugrunde legen, wenn man Lösungen der FM bei (wenn auch niedrigen) endlichen Konzentrationen betrachtet; in diesem Fall ist die athermische Lösung das Modell, von welchem man auszugehen hat.

${ }^{48} \mathrm{H}$. S t a u din ger u. Kl. Fis cher, J. prakt. Chem. 157, 19 [1941]. 